\title{
Review
}

\section{Antithrombin deficiency and its laboratory diagnosis}

\author{
László Muszbek ${ }^{1,2, *}$, Zsuzsanna Bereczky ${ }^{1,2}$, \\ Bettina Kovács ${ }^{3}$ and István Komáromi ${ }^{2}$ \\ ${ }^{1}$ Clinical Research Center, University of Debrecen, \\ Medical and Health Science Center, Debrecen, Hungary \\ ${ }^{2}$ Thrombosis, Haemostasis and Vascular Biology Research \\ Group of the Hungarian Academy of Sciences, University \\ of Debrecen, Medical and Health Science Center, \\ Debrecen, Hungary \\ ${ }^{3}$ Borsod Abaúj Zemplén County and Teaching Hospital, \\ Miskolc, Hungary
}

Keywords: amydolytic assay; antithrombin, antithrombin deficiency; thrombophilia.

\section{Introduction}

The stepwise discovery of antithrombin (AT, SERPINC1) is an exciting story. A detailed account and chronology of the most important events leading to the foundation of our present knowledge about this essential inhibitor of blood coagulation has recently been in an excellent review by Abildgaard (1), recommended for readers interested in this subject. Here, only the issue of nomenclature is mentioned. In the earlier literature, AT used to be termed antithrombin III to distinguish the protein from other proteins with antithrombin activity. Antithrombin I is the thrombin absorbing capacity of fibrin (2) and antithrombin II, more frequently termed heparin cofactor II (SERPIND1) which is another inhibitor of thrombin in plasma (3). Although this is not the only antithrombin present in plasma, we adopted the current nomenclature and use the terms antithrombin and AT throughout the article.

AT is a single-chain glycoprotein with a molecular mass of 58,200 Da. The mature protein consists of 432 amino acids with three internal disulfide bonds. As much of the literature and the existing databases use traditional amino acid residue numbering, starting with the $\mathrm{N}$-terminal residue of the mature protein, we used this system in the article. The numbering system recommended by the Human Genome Variation Society (HGVS) starts with the initiator methionine. In the case of AT, HGVS numbering can be calculated by adding 32, corresponding to the 32 amino acid residues of the leader sequence, to the traditional number. AT has two isoforms which differ only in the extent of glycosylation (4, $5)$. The $\alpha$ isoform is $\mathrm{N}$-glycosylated on four Asn residues (95, 135, 155 and 192), while the $\beta$ isoform lacks glycosylation on Asn135. The $\alpha$ variant is the major AT isoform $(90 \%-95 \%)$ in the circulation, the $\beta$ isoform represents only $5 \%-10 \%$ of AT in plasma. AT is synthesized in the liver, its half-life in the circulation is approximately 2.4 days. A prominent feature of AT is its high affinity binding to negatively charged glycosaminoglycans (GAGs) such as heparin or heparan sulfate which contain specific pentasaccharide units. Due to the lack of carbohydrate residue on Asn135, the $\beta$ isoform binds to GAGs with higher affinity. Heparan sulfate in the form of heparan sulfate proteoglycane (HPSG) is present on the surface of vascular endothelium. Thus, a higher portion of the $\beta$ isoform becomes cleared from the circulation and targets the vessel wall.

\begin{abstract}
*Corresponding author: László Muszbek, MD, PhD, Clinical Research Center, University of Debrecen, Medical and Health Science Center, 98, Nagyerdei krt., Debrecen, 4032, Hungary Phone: +36 52431 956, Fax: +36 52340 011, E-mail: muszbek@med.unideb.hu

Received July 30, 2010; accepted September 25, 2010;

previously published online November 10, 2010

be useful in the practice of routine diagnosis thrombophilia.

Clin Chem Lab Med 2010;48:S67-78.

Antithrombin (AT) belongs to the serpin family and is a key discussed critically, and test results expected in vario vided with a clinically oriented algorithm for the correct
\end{abstract}




\section{The structure of antithrombin and structural changes during its interaction with active clotting factors}

The atomic 3D structure of native AT was resolved approximately 15 years ago $(6,7)$. Since then, numerous AT structures have been published which have helped in the understanding of how AT exerts its inhibitory function (8-14). AT belongs to the family of serine protease inhibitors (serpins), the largest family of protease inhibitors that consists of over 1500 members $(4,14,15)$. These are single chain globular proteins that consist of 300-500 amino acid residues and show about $30 \%$ sequence identity. Serpins share a common tertiary structure; they contain three $\beta$-sheets (A-C) and eight to nine $\alpha$-helices (A-I). A flexible peptide loop, reactive center loop (RCL) containing the reactive site, is exposed on the top of the molecule. RCL contains a sequence which is complementary to the active site of the target protease. All serpins feature significant structural flexibility, which allows dramatic structural changes upon reaction with the protease to be inhibited. These are so-called suicide inhibitors. The target protease cleaves a scissile bond in RCL and then it remains covalently linked to the inhibitor.

Antithrombin is a misnomer, the inhibitory effect of AT is not restricted to thrombin. It is a polyvalent serpin that also inhibits activated factor X (FXa) and to a lesser extent a whole series of serine proteases involved in the hemostatic machinery, including FIXa, FXIa, FXIIa, plasmin and kallikrein $(4,5)$. AT inactivates FVIIa only when it is bound to tissue factor (16-18). AT is a co-called progressive inhibitor; the rate of its reaction with the active coagulation factors is slow, but in the presence of heparin or HPSG, the rate of inhibition is accelerated 500-fold.

AT has a typical serpin secondary and tertiary structure. It consists of nine helices and three $\beta$-sheets (Figure 1). In the uncleaved form, it can exist in two main conformational states. In the native uncleaved form, the 24-membered RCL with the scissile P1-P1' (Arg393-Ser394) bond is outside the main body of AT (Figure 1A). In the latent conformation, the RCL is inserted into the $\beta$-sheet. The latter conformation is thermodynamically more stable than the native form, which is kinetically "trapped" in a high energy state. AT circulates primarily in this kinetically trapped native form. The X-ray structure of this conformation revealed that the P1 residue (Arg393) points to the surface of the body of AT, and the P14-P15 residues are inserted into $\beta$-sheet A, which constrains the RCL and allows contacts between the P1 arginine side chain and the body of AT. Having such a rigid conformation of RCL, AT is a poor inhibitor of FXa or thrombin, and is unable to inhibit FIXa.

The binding of pentasaccharide or heparin containing the pentasaccharide unit causes remarkable changes of the conformation of RCL and its close proximity (Figure $2 \mathrm{~B}$ ). The entrapped part of RCL is expulsed from $\beta$-sheet $A$, the end of the third $\beta$-strand of $\beta$-sheet A moves closer to the fifth $\beta$-strand and helix D becomes elongated. The interaction between AT and the pentasaccharide unit takes place in two steps (not shown on Figure 2); an initial weak binding inter- mediate becomes transformed into a high binding state with 1000 -fold higher affinity. The latter conformation is necessary for the effective formation of the Michaelis complex (Figure 2C) between AT and FXa or FIXa, in which Arg393 of AT and the region in its immediate vicinity is recognized by the protease as a substrate loop. The mechanism of Michaelis complex formation between thrombin and AT is somewhat different. In this case, the conformational change induced by the allosteric effect of pentasaccharide is not sufficient, and probably not even required. Thrombin also binds to heparin, and the bridging effect of heparin of 18 saccharide units or longer, which brings thrombin and AT together, is essential for effective interaction $(11,22,23)$.

After the rate-controlling Michaelis complex formation, the inhibition of active coagulation factors follows the general scheme of serpin action. In the first step of proteolytic reaction, an acyl-enzyme intermediate is formed through an ester bond between Arg393 and the active site serine of the protease. AT undergoes a rapid, drastic and irreversible conformational change where the P14-P3 part of RCL becomes incorporated into $\beta$-sheet $\mathrm{A}$ as an additional strand (Figure 2D), and AT assumes a cleaved relaxed form. This process is accompanied by a 1000-fold reduction in heparin affinity and by a large-scale conformational change in the acylenzyme complex. The protease which is covalently tethered to Arg393 becomes transported from the top to the bottom of AT, approximately $70 \AA$ away from its original position. Due to the distortion of the active site of the protease, the acyl intermediate becomes stabilized and the second step of proteolytic reaction, the release of the cleaved peptide, cannot take place. In this process the protease structure becomes disrupted and the catalytic triad distorted. Only very slow release of the inactive inhibitor and enzyme can be detected from the AT-protease complex (24).

\section{The role of antithrombin in the regulation of coagulation}

AT serves as a highly important regulator of hemostasis; its absence is incompatible with life (5). The primary actions of AT are the inhibition of thrombin mediated fibrin clot formation and the generation of thrombin by FXa. As mentioned earlier, AT also inhibits activated clotting factors higher up in the intrinsic (FIXa, FXIa, FXIIa) and extrinsic (FVIIa-tissue factor complex) pathways. It also inhibits a series of other non-coagulant effects of these clotting factors, including platelet activation, vascular cell signaling, proliferation, cytokine production, etc. There are two paradoxes concerning the effect of AT and its importance in the regulation of clotting machinery: 1) it is a weak progressive inhibitor of activated clotting factors, 2) it fails to inhibit effectively fibrin-bound thrombin and FXa present in an activation complex on the platelet surface.

As discussed in the previous section, interaction with heparin, or its in vivo "substitute" HSPG, significantly accelerates the inhibitory action of AT and makes it a highly effective inhibitor of thrombin, FXa and other active clotting 

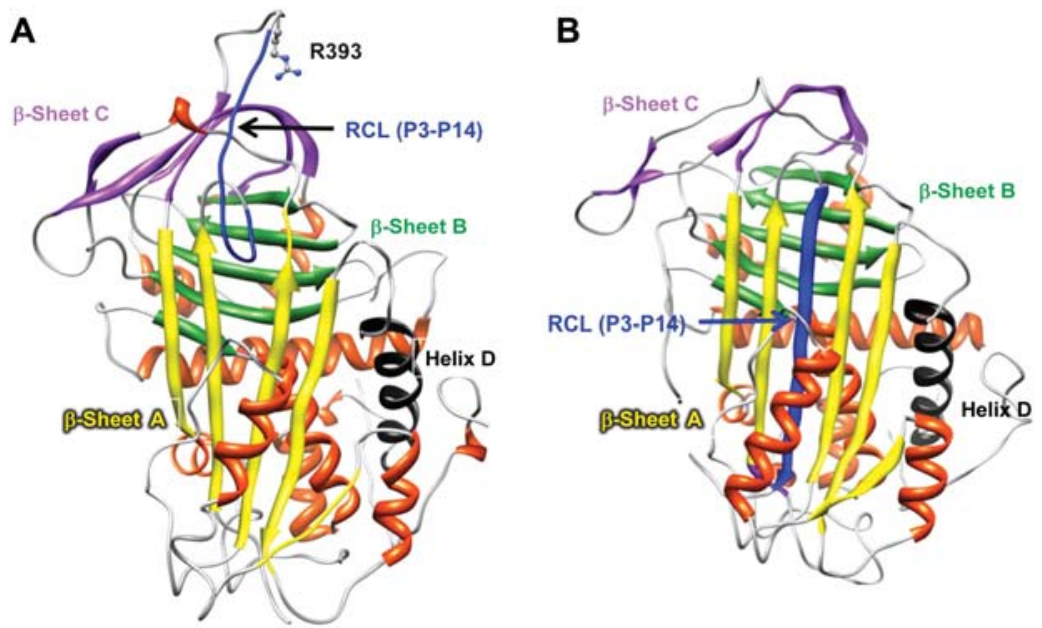

Figure 1 Structural elements of antithrombin (AT) in its native (A) and latent (B) state based on the X-ray structure in the RCSB protein data bank (pdb ID: 2b4x).

The $\beta$-sheets A, B and C are colored yellow, green and purple, respectively. Helical secondary structural elements are shown in orange, except for helix D that is shown in black. Helix D plays a crucial role in heparin pentasaccharide binding. (In the case of serpins, conserved helical structure elements are identified by capital letters.) The P3-P14 portion of the reactive center loop consisting of P1-P17 and P1'-P17' residues is colored dark blue. The Arg393 (P1) residue is shown by a ball-and-stick representation. In the latent state, a substantial portion of the reactive center loop is inserted into $\beta$-sheet $\mathrm{A}$ as an additional $\beta$-strand. The figures were prepared using Chimera software (Resource for Biocomputing, Visualization, and Informatics at the University of California, San Francisco, CA, USA) (19).

factors (5). HSPG is widely available on the vascular endothelium and/or in the underlying subendothelial matrix and tissues. Only a minor portion of HSPG contains the 3-O- sulfated pentasaccharide unit required for the acceleration of AT activity (25). This active HSPG (A-HSPG) species constitutes $5 \%$ of the total HSPG associated with rat microvas-

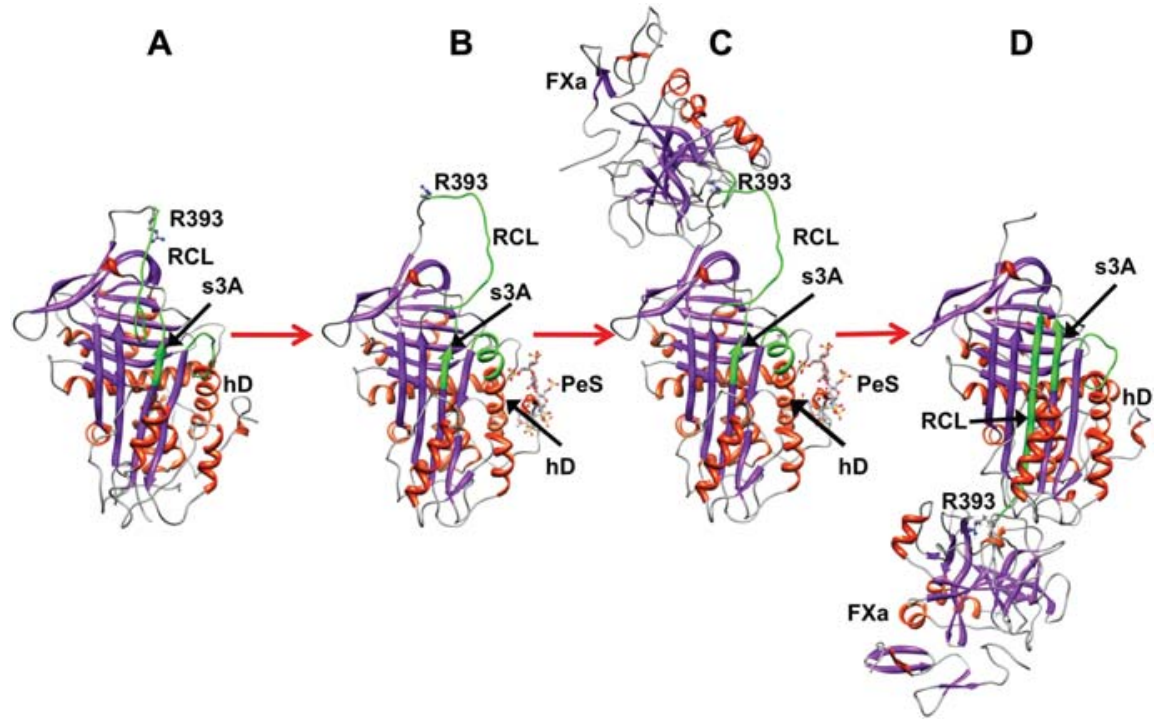

Figure 2 Schematic representation of the mechanism of activation and action of antithrombin (AT) on FXa.

(A) The native (circulating) form of AT, (B) the pentasaccharide-activated AT, (C) AT-factor Xa (FXa) complex; only epidermal growth factor 2 (EGF 2) and serine protease domains of FXa are shown, (D) the acyl-enzyme complex in which FXa is covalently linked to AT. For the A, B and C parts of the figure the X-ray coordinates [pdb IDs are 2b4x, 2gd4 (AT only) and 2gd4 (AT-FXa), respectively] deposited in the protein data bank (20) were used. The construction of (D) was based on the structure of the $\alpha_{1}$-antitrypsin-elastase acyl-enzyme complex (pdb ID: 2d26) (21). $\alpha_{1}$-antitrypsin was replaced by AT and elastase was substituted by FXa. Next, energy minimization of the constructed complex was performed with Yasara software (Yasara Biosciences GmbH, Vienna, Austria) (www.yasara.org). $\beta$-sheets and helices are shown in magenta and orange, respectively. Peptide sections that undergo remarkable conformational changes [P1-P15 section of the reactive center loop (RCL), the third $\beta$-strand of $\beta$-sheet $\mathrm{A}(\mathrm{s} 3 \mathrm{~A})$ and helix $\mathrm{D}(\mathrm{hD})]$ are depicted in green. The Arg393 residue and the pentasaccharide $(\mathrm{PeS})$ are shown by ball-and-stick representations. The figures was prepared using Chimera software (19). 
cular endothelial cells. Studies of its distribution in different vessels $(26,27)$ suggest that A-HSPG present on the surface of endothelial cells provides a basic level of activated AT for anticoagulant activity, while the availability of a much larger pool following endothelial damage and vessel wall injury dramatically increases the anticoagulant potential of AT. The relative importance of progressive AT activity or AHSPG induced activity in the physiological regulation of blood coagulation is not clear. The fact that homozygous mutations at the heparin binding site, which render AT unable to bind GAGs, are compatible with life (see later for details), as opposed to other homozygous AT deficiencies, suggests the importance of progressive activity. On the other hand, the severe thrombophilia seen in such patients underlines the importance of heparin/HSPG induced AT activation.

The association of active clotting factors with the surface of activated platelets and with the fibrin clot significantly modifies the inhibitory effect of AT or AT-heparin. When present in the prothombinase complex on platelets or phospholipid surfaces, FXa escapes the inhibition by AT (28-30). Thrombin bound to fibrin or to fibrin degradation products becomes refractive to inhibition by the AT-heparin complex. Fibrin, heparin and thrombin form a ternary complex, in which thrombin exosite 1 and exosite 2 are occupied by fibrin and heparin, respectively (31). This prevents AT-associated heparin from interacting with exosite 2 on fibrinbound thrombin, and the bridging action of heparin cannot operate. It is of interest that FVIIa becomes sensitive to inhibition by AT only when bound to tissue factor $(16,17)$. The above findings suggest a double role for the AT and AT-AHSPG complex in the physiological regulation of blood coagulation. It might control low-level thrombin formation that occurs physiologically in the unperturbed circulation by the inhibition of tissue factor-FVIIa complex, FXa and perhaps other active clotting factors. AT might also exert a scavenger function by neutralizing FXa and thrombin that have escaped from the clot and from the activation complex. The latter mechanism could prevent the propagation of the clot to areas away from the site of vascular injury.

In vivo, the AT-protease complex is rapidly eliminated from the circulation by utilizing a common serpin-protease complex clearance pathway. Binding to members of the lowdensity lipoprotein receptor family, primarily to low density lipoprotein-related protein which is an important receptor in the liver, is the main pathway of the elimination of serpinprotease complexes $(5,32)$.

\section{Gene structure of antithrombin}

The gene for human AT (SERPINC1) is located at the 1q23q25 position and contains seven exons producing a 1.4-kb messenger RNA (mRNA), and six introns $(33,34)$. All the exon/intron boundaries follow the GT-AG rule. Nine complete and one partial Alu repeats were identified in introns $1,2,3 \mathrm{~B}, 4$ and 5 . There is a highly polymorphic trinucleotide repeat sequence in intron 4 which is useful for haplotype analysis in studies of recurrent mutations and for linkage analysis in families with thrombosis (35). Primer extension analysis has mapped the AT transcriptional start site in liver cells to a position $72 \mathrm{bp}$ upstream of the ATG translation initiator codon (36). A leader sequence of 32 amino acids is encoded by exon 1 and the 5' end of exon 2 (37). The heparin binding site of AT is encoded by exon 2 and exon $3 \mathrm{a}$. The reactive site, located in the carboxy-terminal part of the protein, is encoded by exon 6 .

\section{Epidemiology of antithrombin deficiency}

The prevalence of inherited AT deficiency in the general population is estimated to be between 1:2000 and 1:3000 (38). Most of the genetic defects result in type II (qualitative) deficiencies (39). The prevalence of AT deficiency in patients with venous thromboembolism (VTE) is much higher, between 1:20 and 1:200 (40). According to an Italian study of symptomatic patients and relatives, type I mutations (quantitative deficiencies) are more frequent than type II variants (41).

In unselected patients with a history of VTE, the frequency of AT deficiency is $0.5 \%-1.1 \%(40,42)$. In a cumulated analysis of 1705 selected patients with VTE, the frequency of AT deficiency was $2.4 \%$ (43). During a mean follow-up time of 2.3 years the incidence of venous thrombosis was high; being $12 \%$ in individuals with hereditary AT deficiency in a small Italian cohort (44). For comparison, the incidence of thrombosis in protein C (PC) and protein S (PS) deficiency was $2.8 \%$ and $3.3 \%$, respectively.

In the large prospective EPCOT study (European Prospective Cohort on Thrombophilia), the risk of first VTE in asymptomatic AT, PC or PS deficient individuals and in individuals with Factor $\mathrm{V}$ Leiden mutation was analyzed $(n=575)$. During the 5.7 year of follow-up, $4.5 \%$ of these individuals developed VTE, the annual incidence of first VTE was the highest in those with AT deficiency (1.7\%/ year) (45). Based on the prevalence data in the general population and in VTE patients, the relative risk of VTE in patients with AT deficiency was estimated to be approximately 25-50-fold (46). Since then, prospective and casecontrol studies have calculated the same magnitude of VTE risk conferred by AT deficiency in different ethnical groups (47-49). Based on the results of these epidemiological studies, it can be concluded that the risk of VTE conferred by hereditary AT deficiency is the highest among inherited thrombophilias. However, the risk of VTE seems to vary according to the subtypes of AT deficiency (see later).

AT deficiency also represents an increased risk for development of PE in deep venous thrombosis (DVT), and an increased risk for recurrence of VTE. In an Italian study of patients with proximal DVT, the risk of pulmonary embolism (PE) was 2.4-fold (95\% CI: 1.61-3.63) in AT deficient patients compared to individuals who developed DVT without inherited thrombophilia (50). In AT deficiency, the annual incidence of recurrent VTE was found to be $10 \%(95 \%$ CI: $6.1 \%-15.4 \%$ ) in a recently released Dutch study (51). In 
an Italian cohort, the adjusted hazard ratio for the recurrence of VTE was 1.9 (95\% CI: 1.0-3.9) (52).

\section{Molecular genetic background of antithrombin deficiency, genotype-phenotype correlations}

The first report on AT deficiency was described by Egeberg in 1965 (53). The first functional AT defect, AT Budapest, was reported by Sas et al. in 1974 (54). Since then, a high number of deficient patients have been identified, and the molecular genetic background was clarified in a significant number of cases. According to the recommendations of the International Society on Thrombosis and Haemostasis, AT deficiency is classified as type I (quantitative) and type II (qualitative) deficiency (55). In type I deficiency, AT activity and the antigen concentration are equally decreased, suggesting defective synthesis or secretion of the protein. In type II deficiency, the defect may involve the reactive site (type II RS), the heparin-binding site (type II HBS) or it can exert a pleiotropic effect (type II PE) (56). The inheritance of AT deficiency, in general, is autosomal dominant. However, in the case of type II HBS deficiency, it often shows incomplete penetrance or an autosomal recessive pattern. The majority of AT deficient patients are heterozygous for the defect with typical AT activity values approximately 50\%. Homozygosity is incompatible with life, with the exception of type II HBS variant (described later). The molecular genetic background of AT deficiency is heterogeneous. The mutations are best summarized in the Antithrombin Mutation Database (http://www1.imperial.ac.uk/medicine/about/divisions/departmentofmedicine/experimentalmedicine/haematology/coag/antithrombin/) and in the database of human gene mutation data (HGMD) (http://www.hgmd.cf.ac.uk) (Figure 3).

Almost $50 \%$ of the 215 different mutations that have been reported in the HGMD are missense mutations. Small deletions and insertions are also common, contributing $20 \%$ and $10 \%$, respectively. Non-sense mutations and splicing site mutations represent $8 \%$ and $5 \%$ of all reported causative sequence variants, respectively. Whole or partial gene deletions are relatively frequent $(5 \%)$, while complex rearrangements are rare.

Type I AT deficiencies are most commonly caused by insertions or deletions leading to frameshift and premature stop codon, or less commonly by non-sense mutations. These mutations obviously explain the type I phenotype, primarily a result of unstable mRNA transcripts and/or the presence of truncated proteins. Large gene segment deletions also lead to type I deficiency. By screening "mutation negative" AT deficient cases using the multiplex ligation-dependent probe amplification (MLPA) technique, several gross deletions were identified. The breakpoints are often located within Alu repeat elements (57). Amino acid changes caused by single nucleotide substitutions within the coding region of SERPINC1 may also lead to type I deficiency. In this case, the absence of mutant protein in the circulation is due to misfolding or a secretion defect (4).
The type II AT deficiencies are most commonly caused by missense mutations. Among the mutations known to involve the reactive site domain, two regions are preferred: the hinge region (most frequently residues Ala382 and Ala384) and around the reactive domain at residues Gly 392 (AT Stockholm), Arg393 and Ser394 (58). Most of the missense mutations leading to type II HBS deficiency affect residues Pro41 (AT Basel), Arg47 (AT Padua I), Leu99 (AT Budapest 3) and Arg129. Practically all patients with AT Budapest 3 (p.Leu99Phe) mutation described to date were of South Eastern European origin, which may suggest a founder effect $(35,59-61)$. Type II PE deficiency is caused by mutations involving residues 402, 404-407 and 429. This region is responsible for both the structural and functional integrity of AT. These mutations lead to impaired function of the reactive site and also to reduced secretion (58). A new pleiotropic mutant (AT Murcia, p.K241E) has been described recently in which altered glycosylation of the molecule led to impaired heparin binding and thrombin inhibition (62). Some of the missense mutations occurred in the mobile regions of AT, mainly at the hinges of the reactive center loop, or in the region involved in the shutter-like opening of the main $\beta$-sheet of the molecule. The latter is required for insertion of the reactive loop into the $\beta$-sheet. Even change in a single amino acid in these sensitive regions can lead to conformational changes, loss of stability that facilitates the formation of intermolecular linkages and lead to the formation of oligomers or transformation to the latent conformation (4). The secretion of these variants is also impaired which results in a circulating deficiency.

Homozygous type I AT deficiency is not compatible with life and heterozygous patients usually suffer severe thrombosis at a young age. The same stands for type II RS and type II PE deficiencies. However, there is at least one notable exception. The heterozygous p.Ala384Ser mutation (AT Cambridge II) causes type II RS deficiency with a mild phenotype, and this mutation can also exist in homozygous form $(63,64)$. Type II HBS deficiency confers a lower risk of thrombosis compared with the other subtypes $(65,66)$. Homozygous type II HBS patients usually survive, thrombosis may develop even earlier (frequently in childhood) than in patients with heterozygous type I or other type II deficiencies.

Symptoms of AT deficiency are DVT and/or PE which are often recurrent. DVT not infrequently develops at unusual sites, such as in the proximal extremities, and in mesenteric, renal, portal, retinal and cerebral veins (67-70). Intracardial atrial thrombosis has also been reported (71). The risk of thrombosis conferred by AT deficiency to pregnant women is significantly greater than in other deficiencies. The estimated risk is 1:2.8 for women with type I deficiency, which is approximately 350 -times higher than the risk conferred by pregnancy alone $(72,73)$.

In addition to venous thrombosis, occasionally, arterial thrombosis has also been reported in patients with AT deficiency $(74,75)$. AT Cambridge (p.A384S) mutation increased the risk of myocardial infarction 5.66-fold as reported by a Spanish study that enrolled 1224 patients and 


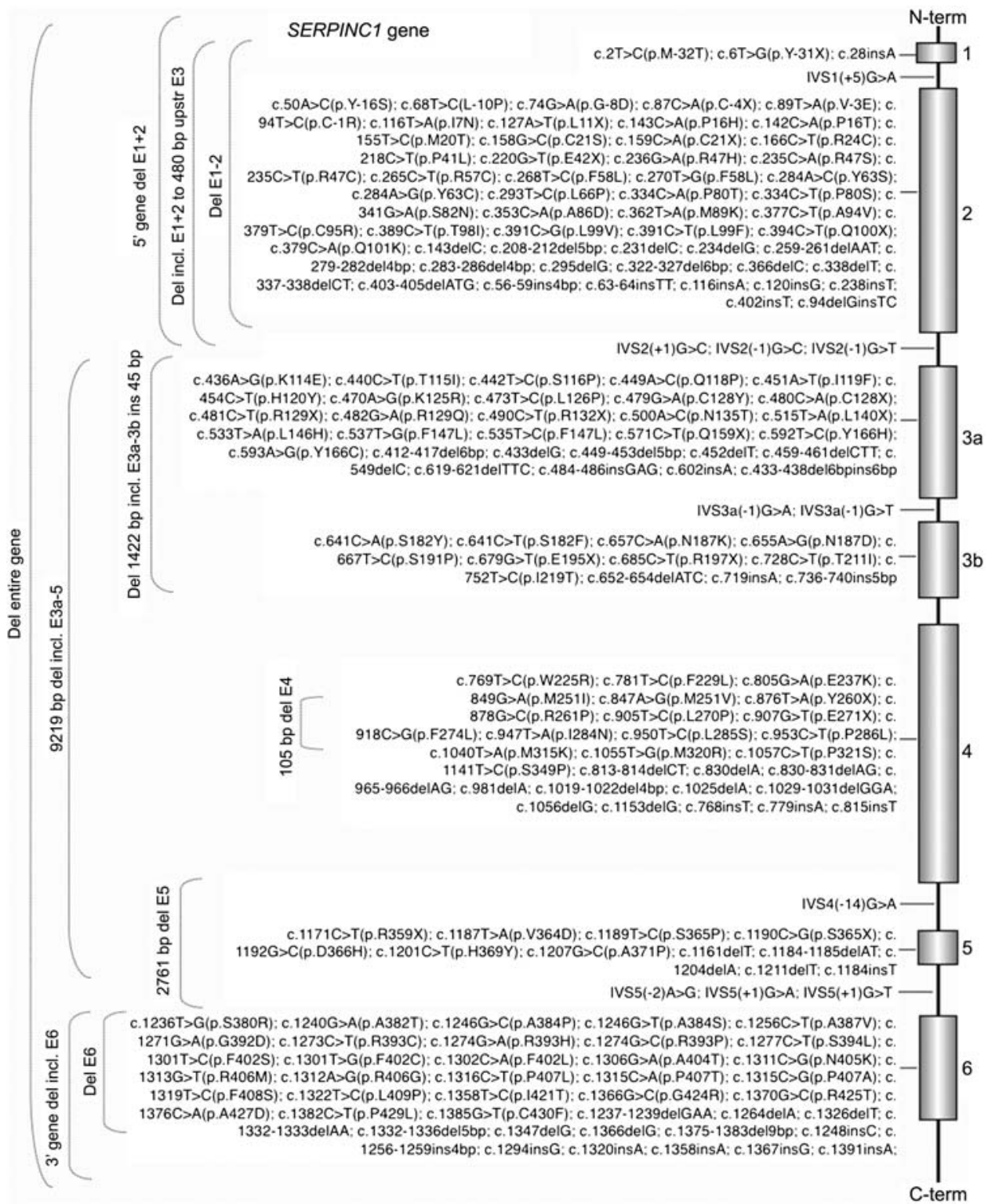

Figure 3 Distribution of causative mutations in the SERPINC1 gene according to the database of human gene mutation data (HGMD) that have been published to date (www.hgmd.cf.ac.uk).

Nucleotide numbering is given relative to the first nucleic acid of the initiator ATG codon. Amino acids are numbered according to the mature protein, where the first methionine is numbered -32 .

1649 controls (76). In contrast, in a large cohort of relatives of VTE patients with PC, PS or AT deficiency $(n=468)$, the risk of arterial thrombosis in those $<55$ year of age was increased only in PC and PS deficient patients, while AT deficiency was not associated with an increased risk (hazard ratio $1.1,95 \% \mathrm{CI}$ : $0.1-10.9)$ (77). According to a recent meta-analysis of studies involving children with arterial ischemic stroke and cerebral venous sinus thrombosis, the summary OR for the risk of arterial ischemic stroke in children having AT deficiency was 3.29 (95\% CI: 0.70-15.48), while the OR for the risk of cerebral venous sinus thrombosis was 18.41 (95\% CI: 3.25-104.29) (78). These findings do not support a significant contribution of AT deficiency to the risk of atherothrombotic events.

\section{Acquired antithrombin deficiency}

In healthy full-term newborns, the concentration of AT is in the range of $51 \%-75 \%$ of adult average values (79). Due to severe immaturity of the liver, in preterm infants AT concentrations can be much lower than in full terms infants (80). AT concentrations reach adult ranges by the age of 1 year (81). Production of AT is reduced in liver disease with impaired hepatic function. In patients with nephrotic syndrome or other diseases associated with renal or enteral protein loss, the low AT concentration is due to increased elimination. Low concentrations of AT as a result of consumption are found in patients with sepsis, disseminated intravascular coagulation, large thrombus, thrombotic micro- 
angiopathy, acute hemolytic transfusion reactions and malignancies (82). There are two notable examples of druginduced AT deficiency. Long-term therapy with unfractionated heparin is a common cause of moderate AT consumption, which is probably the result of greatly enhanced formation of thrombin-AT complex in plasma. Therapy with L-asparaginase leads to intracellular retention of AT within the endoplasmic reticulum, perhaps due to interference with folding of the molecule and with glycosylation of the protein $(83,84)$. Interestingly, co-administration of dexaamethasone with L-asparaginase increased the concentration of AT and reduced the risk of thrombosis. The effect of dexamethasone is possibly due to induced expression of heat shock proteins and endoplasmic reticulum-associated chaperons which prevent the conformational effect of L-asparaginase (85).

\section{Laboratory diagnosis of antithrombin deficiency}

A first-line test for the diagnosis of AT deficiency should detect all deficiencies, i.e., AT deficiencies due to decreased AT concentration as well as to a defective molecule. Therefore, the first line test should be a functional assay. The original clotting methods where the inhibition of thrombin by diluted native serum or defibrinated plasma was measured by fibrinogen clotting are impractical and inaccurate and not in use any longer. With the modern chromogenic (amidolytic) assays, the inhibition of thrombin or FXa activity by AT is measured using thrombin/FXa specific tri, or tetra-peptide substrates which show sequential similarity to the P1-P3 or $\mathrm{P} 1-\mathrm{P} 4$ sequences of the natural substrates of these enzymes (Figure 4) $(86,87)$. The peptides conform to the active site of the respective active clotting factor and a para-nitroanaline (pNA) group is attached to their C-terminal end. Thrombin or FXa rapidly release the pNA group from their peptide substrate. Free pNA, as opposed to the peptide-bound form,

$$
\begin{aligned}
& \text { Heparin + AT } \rightarrow \text { heparin-AT* complex } \\
& \text { Heparin-AT* }+\frac{\text { thrombin (in excess) }}{\mathrm{FXa} \text { (in excess) }} \rightarrow \\
& \rightarrow \text { heparin-AT*-thrombin+thrombin (residual) } \rightarrow \\
& \rightarrow \text { heparin-AT*-FXa+FXa (residual) } \rightarrow \\
& \rightarrow \mathrm{R}(\text { thr })-\mathrm{pNA}+\text { thrombin } \rightarrow \mathrm{R}(\text { thr })-\mathrm{COOH}+\mathrm{pNA} \\
& \rightarrow \mathrm{R}(\mathrm{FXa})-\mathrm{pNA}+\mathrm{FXa} \rightarrow \mathrm{R}(\mathrm{FXa})-\mathrm{COOH}+\mathrm{pNA} \\
& \text { Spectrophotometric measurement at } 405 \mathrm{~nm} \\
& \text { R(thr): H-D-Phe-Pip-Arg } \\
& \text { R(FXa): Ac-D-Arg-Gly-Arg }
\end{aligned}
$$

Figure 4 Measurement principle of chromogenic antithrombin assays.

Both anti-thrombin and anti-FXa heparin cofactor assays are demonstrated. AT, antithrombin; $\mathrm{AT}^{*}$, antithrombin activated by heparin; FXa, activated factor $\mathrm{X}$; R, the peptide part of chomogenic thrombin or FXa substrates; pNA, para-nitro aniline. In the last line, the oligopeptide components of a thrombin (thr) substrate (S-2238) and a FXa substrate (S-2772) are shown. has strong light absorption at $405 \mathrm{~nm}$ and its release can be easily monitored spectrophotometrically. The assays can be performed in the presence of heparin (heparin cofactor activity) or without heparin (progressive activity). In the former assays, the inhibition of active clotting factors is very quick, while in the latter cases more time is required for ATII to exert its inhibitory action. As only the heparin cofactor activity is decreased in all subtypes of AT deficiency (Table 1), the assay measuring this activity is the generally accepted first line test for the diagnosis of deficiency. Unfortunately, as external quality control exercises reveal, the improper practice of using only an antigenic AT assay, which detects $<50 \%$ of AT deficiencies, still exists in a few laboratories.

Figure 4 demonstrates the assay principle of amidolytic AT assays. Heparin binds to AT making it highly reactive with thrombin and FXa (activated AT; AT*). Thrombin or FXa is added in excess of AT and a part of it becomes rapidly complexed with heparin-AT*, in the complex AT activity is abrogated. The extent of thrombin/FXa inhibition depends on plasma AT activity, and the residual free thrombin or FXa is inversely related to AT activity. The amount of free thrombin or FXa is measured using a chromogenic substrate described above. The increase in absorbance at $405 \mathrm{~nm}$ can be measured using a kinetic or end-point method (the former is preferred), and the change of absorbance is converted to AT activity using a calibration curve. Reference plasma of known AT activity is used to construct the calibration curve. A WHO international standard (2nd International Standard Antithrombin, Plasma, NIBSC code: 93/768) with an assigned potency of 0.85 International Units (IU) is available from the National Institute for Biological Standards and Control (NIBSC; Potters Bar, UK). This international plasma standard should be used by companies for the calibration of their reference plasma and this information should be stated on the application sheet.

The chromogenic heparin cofactor AT assay has good reproducibility. Laboratories equipped with automated laboratory analyzers or coagulometer should aim for within-batch precision $\mathrm{CV} \leq 2 \%$, and for within-laboratory reproducibility (total error) $\mathrm{CV} \leq 5 \%$. According to a previous report based on the quality assessment program for thrombophilia screening by the ECAT Foundation which included 136 laboratories during the time period 1996-2001, the median long-term within-laboratory analytical CV was $7.6 \%$ with a $95 \% \mathrm{CI}$ of $3.6-35.5$ (88). These values suggest that for many laboratories, there is much work needed to improve the quality

Table 1 Laboratory diagnosis and classification of antithrombin (AT) deficiencies.

\begin{tabular}{llll}
\hline $\begin{array}{l}\text { Subtypes of AT } \\
\text { deficiencies }\end{array}$ & $\begin{array}{l}\text { Heparin cofactor } \\
\text { AT assay }\end{array}$ & $\begin{array}{l}\text { Progressive } \\
\text { AT assay }\end{array}$ & $\begin{array}{l}\text { AT antigen } \\
\text { assay }\end{array}$ \\
\hline Type I & $\downarrow$ & $\downarrow$ & $\downarrow$ \\
Type II RS & $\downarrow$ & $\downarrow$ & $\mathrm{n}$ \\
Type II HBS & $\downarrow$ & $\mathrm{n}$ & $\mathrm{n}$ \\
Type II PE & $\downarrow$ & $\downarrow$ & $\mathrm{n}$ or subnormal \\
\hline
\end{tabular}

RS, reactive site; HBS, heparin binding site; PE, pleiotrop; $\mathrm{n}$, normal. 
performance of AT assays. Joining an international accredited external quality assessment program is highly recommended for laboratories routinely performing AT measurements.

Human thrombin was used in previous thrombin inhibition assays. Human thrombin also reacted with heparin cofactor II and made the assay relatively insensitive for the detection of AT deficiency $(89,90)$. In most commercial kits, human thrombin has been replaced by bovine thrombin which shows minimal reaction with heparin cofactor II. FXa does not react with heparin cofactor II at all. Heparin cofactor AT assays based on bovine thrombin and FXa inhibition seem to function equally well, the sensitivity of both assays is close to $100 \%$. As the reactive site of thrombin and FXa differs somewhat, one would expect that AT deficiencies caused by certain mutations around the reactive site are detected by the two types of assays with different sensitivity. Indeed, the Ala384Ser mutation (AT Cambridge II) which is a relatively prevalent variant in the general population, is not detected by the anti-FXa assay, but anti-thrombin activity is mildly, but significantly, reduced $(63,64)$. However, this mutation causes only a relatively mild thrombophilia, and even elderly homozygous individuals might lack the history of VTE. In contrast, according to our experience with a high number of AT Budapest 3 mutants, the anti-Xa assay is significantly more sensitive in detecting this type II HBS deficiency compared with the assay based on thrombin inhibition.

Progressive AT assays are based on the same principle as heparin cofactor assays, but are performed in the absence of heparin on less diluted plasma samples. In addition, the incubation time is prolonged significantly. Unfortunately, the application sheets for the commercial AT activity assays do not provide a description on how to use the test kit as a progressive assay, the appropriate conditions need to be established by the user. These conditions may vary among commercial kits, and the user needs to experiment. As a starting point, 10-fold diluted plasma and a 15-min incubation time is recommended. Although this test is essential for the diagnosis of type II HBS deficiency (Table 1), for the reasons mentioned above, the progressive assay is very much under utilized in the diagnosis and classification of AT deficiencies (91). As discussed earlier, differentiation between heterozygous type II HBS deficiency causing mild thrombophilia and other type of functional defects causing a severe phenotype is of clinical relevance. In certain, clinical set-ups this might influence the decision concerning anticoagulant therapy. Distinguishing between homozygous type II HBS deficient patients who have a very severe phenotype, and heterozygotes is also of clinical relevance.

Measurement of AT antigen concentrations is required for the classification of AT deficiencies. Traditional electroimmunodiffusion and radial-immunodiffusion techniques are too time consuming, imprecise and their use is no longer recommended. At present, latex-enhanced immuno nephelometry is the most frequently used method for measurement of AT antigen concentrations (92), and commercial kits for this purpose are available.

It is rather surprising that no reference interval determined according to guideline (C28-A3) from the Clinical and Lab- oratory Standards Institute (CLSI; Wayne, PA, USA) is available for AT activity and antigen. A number of different "normal" ranges, varying within a narrow interval have been reported in the literature and are available in manufacturer's application sheets. Accepting $80 \%$ of the average nor$\mathrm{mal}(0.8 \mathrm{IU} / \mathrm{mL})$ as the lower limit of reference interval for AT activity seems to be an acceptable compromise, and most laboratories use this value. The upper limit of the reference interval does not have any clinical relevance. AT antigen concentrations can be expressed as mass concentration $(\mathrm{mg} / \mathrm{L})$, although there are a rather wide variety of "normal" ranges for mass concentration. In addition, values for mass concentration are difficult to compare to AT activity values. For this reason most laboratories use the same principle and the same reference interval for AT antigen as for AT activity. It should be noted that by definition, $2.5 \%$ of the values obtained with normal, non-deficient samples are below the lower limit of the reference interval, and values between $70 \%$ and $80 \%$ should be interpreted with extreme caution.

Platelet poor citrated plasma is used for both activity and antigen measurements and can be stored at $-20^{\circ} \mathrm{C}$ for up to 4 months. Measurement of AT activity and concentration is not recommended within 3 months of an acute event. During this period, if values are within the reference interval, the exclusion of AT deficiency is possible, but the diagnosis of AT deficiency cannot be confirmed. In a number of cases, AT determination is requested for patients who are on anticoagulant therapy. Oral anticoagulant therapy with vitamin $\mathrm{K}$ antagonists, such as warfarin or acenocoumarol might increase the level of AT (93-98), while administration of unfractionated heparin decreases the concentration of AT $(58,94,97,99)$. Low molecular weight heparins do not have such an effect (100). For these reasons, we do not recommend diagnosing AT deficiency in patients who are undergoing unfractionated heparin therapy. Also, we do not recommend attempting to exclude AT deficiency during oral anticoagulant therapy. In our experience switching from oral anticoagulant therapy to low molecular heparin for 10 days prior to blood collection is a good compromise which allows the measurement of valid AT values. The algorithm used in the authors' laboratory for the diagnosis and classification of AT deficiency is demonstrated in Figure 5. If heparin cofactor AT activity is $<80 \%$ we carefully look for and exclude acquired AT deficiencies, such as liver disease, renal or enteral protein loss, consumption coagulopathy, unfractionated heparin treatment or therapy with 1-asparaginase. To establish the diagnosis, repeated tests are required from different blood samples collected from the same person. If feasible, we recommend a time interval of at least 3 weeks between the two blood collections. If the heparin cofactor activity is repeatedly equal to or $<70 \%$ and acquired causes have been excluded, the laboratory diagnosis of inherited AT deficiency can be established. Between $70 \%$ and $80 \%$ of AT activity, it is highly recommended to confirm the diagnosis by molecular genetic testing. Once AT deficiency is diagnosed, the next step is its classification, which occurs in two steps. Plasma AT antigen concentrations are measured to differentiate between type I and type II deficiency. Decreased 


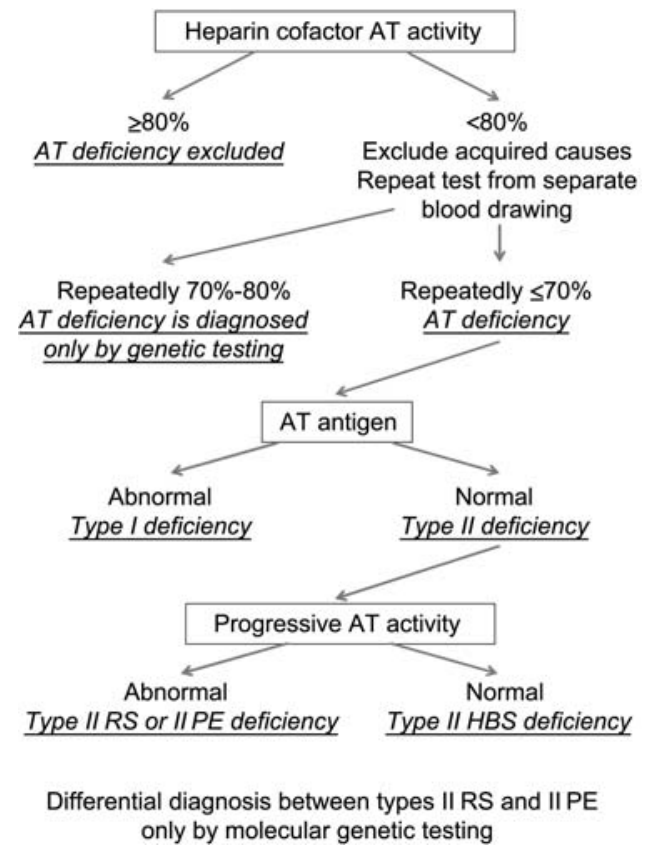

Figure 5 Laboratory diagnosis and classification of antithrombin (AT) deficiency.

Assays are shown in rectangles, diagnoses in italics are underlined. As stated in the text, we do not recommend diagnosing AT deficiency in patients receiving therapy with unfractionated heparin, and do not recommend excluding AT deficiency during oral anticoagulant therapy.

AT antigen implies type I deficiency, while AT antigen in the normal range indicates type II deficiency. Finally, it is clinically important to distinguish II HBS subtype from other type II variants by performing a progressive activity assay. As opposed to other type II subtypes, HBS variants have normal progressive activity. Table 1 summarizes the results of diagnostic and classification tests in different subtypes of AT deficiency.

\section{Concluding remarks}

AT is a slow progressive inhibitor of active clotting factors, particularly thrombin and factor Xa (FXa). Glycosaminoglycans with a 3-O-sulfated pentasaccharide unit, like heparin or heparan sulfate, bind to AT with high affinity and greatly accelerate the reaction with active clotting factors. Even in the presence of heparin/heparan sulfate, AT poorly inhibits FXa in activation complex and fibrin-bound thrombin. It might control low level thrombin formation that occurs physiologically, and could also exert a scavenger function by neutralizing FXa and thrombin that have escaped from the clot and from the activation complex. In general, inherited AT deficiency causes severe thrombophilia. However, the phenotypic appearance varies with different subtypes. Homozygous type I AT deficiency caused by decreased synthesis or secretion is incompatible with life. In the heterozygous form, it is frequently accompanied by DVT, not infrequently of unusual localization, or PE after the second decade of life. Functional defects (type II AT deficiencies) caused by missense mutations are classified according to the site that is affected by the mutation as reactive site (RS), heparin binding site (HBS) AT deficiencies or multiple site defect caused by mutations with pleiotropic effects (PE). Type II HBS subtype is less severe than other AT deficiencies. In the heterozygous form it presents with only mild thrombophilia, and homozygotes also survive, although usually with very early thrombotic complications.

The diagnosis of inherited AT deficiency is important for establishing the risk of recurrent thrombotic events. The diagnosis might influence the clinical decision concerning the duration of anticoagulant therapy. The diagnosis is established by laboratory tests, although the exclusion of acquired deficiency requires careful clinical attention. The first line test is a functional chromogenic heparin cofactor assay, which measures the inhibition of thrombin or the inhibition of FXa in the presence of heparin. Both anti-thrombin and anti-FXa assays perform well and, with very few exceptions, detect all subtypes of AT deficiency. The reference interval is quite narrow and in most cases the range of $80 \%-120 \%$ of average normal is accepted. Measurement of AT antigen concentrations allows differentiation between type I and type II AT deficiencies, while progressive AT activity assays are required to make the clinically important distinction between type II HBS and other type II subtypes. Increased utilization of a progressive activity assay is desirable. The diagnosis might be confirmed by molecular genetic testing, which is important in the case of activities in the range of $70 \%-80 \%$.

\section{Acknowledgements}

This work was supported by a grant from the Hungarian National Research Fund (OTKA K78386).

\section{Conflict of interest statement}

Author's conflict of interest disclosure: The authors stated that there are no conflicts of interest regarding the publication of this article.

Research funding: None declared.

Employment or leadership: None declared.

Honorarium: None declared.

\section{References}

1. Abildgaard U. Antithrombin - early prophecies and present challenges. Thromb Haemost 2007;98:97-104.

2. Mosesson MW. Update on antithrombin I (fibrin). Thromb Haemost 2007;98:105-8.

3. Rau JC, Beaulieu LM, Huntington JA, Church FC. Serpins in thrombosis, hemostasis and fibrinolysis. J Thromb Haemost 2007;5 Suppl 1:102-15.

4. Hernandez-Espinosa D, Ordonez A, Vicente V, Corral J. Factors with conformational effects on haemostatic serpins: implications in thrombosis. Thromb Haemost 2007;98:557-63. 
5. Bock SC. Antithrombin III and heparin cofactor II. In: Colman RW, Clowes AW, Goldhaber SZ, Marder VJ, George JN, editors. Hemostasis and Thrombosis, Vol. Philadelphia: Lippincott, 2006:235-48.

6. Carrell RW, Stein PE, Fermi G, Wardell MR. Biological implications of a 3-A structure of dimeric antithrombin. Structure 1994;2:257-70.

7. Schreuder HA, de Boer B, Dijkema R, Mulders J, Theunissen HJ, Grootenhuis PD, et al. The intact and cleaved human antithrombin III complex as a model for serpin-proteinase interactions. Nat Struct Biol 1994;1:48-54.

8. Whisstock JC, Pike RN, Jin L, Skinner R, Pei XY, Carrell RW, et al. Conformational changes in serpins: II. The mechanism of activation of antithrombin by heparindagger. J Mol Biol 2000; 301:1287-305.

9. Huntington JA, Gettins PG. Conformational conversion of antithrombin to a fully activated substrate of factor Xa without need for heparin. Biochemistry 1998;37:3272-7.

10. Whisstock JC, Bottomley SP. Molecular gymnastics: serpin structure, folding and misfolding. Curr Opin Struct Biol 2006;16:761-8.

11. Johnson DJ, Li W, Adams TE, Huntington JA. AntithrombinS195A factor Xa-heparin structure reveals the allosteric mechanism of antithrombin activation. EMBO J 2006;25:2029-37.

12. Langdown J, Belzar KJ, Savory WJ, Baglin TP, Huntington JA. The critical role of hinge-region expulsion in the induced-fit heparin binding mechanism of antithrombin. J Mol Biol 2009; 386:1278-89.

13. Johnson DJ, Langdown J, Huntington JA. Molecular basis of factor IXa recognition by heparin-activated antithrombin revealed by a 1.7-A structure of the ternary complex. Proc Natl Acad Sci USA 2010;107:645-50.

14. Huntington JA. Shape-shifting serpins - advantages of a mobile mechanism. Trends Biochem Sci 2006;31:427-35.

15. Silverman GA, Bird PI, Carrell RW, Church FC, Coughlin PB, Gettins PG, et al. The serpins are an expanding superfamily of structurally similar but functionally diverse proteins. Evolution, mechanism of inhibition, novel functions, and a revised nomenclature. J Biol Chem 2001;276:33293-6.

16. Rao LV, Rapaport SI, Hoang AD. Binding of factor VIIa to tissue factor permits rapid antithrombin III/heparin inhibition of factor VIIa. Blood 1993;81:2600-7.

17. Lawson JH, Butenas S, Ribarik N, Mann KG. Complexdependent inhibition of factor VIIa by antithrombin III and heparin. J Biol Chem 1993;268:767-70.

18. Broze GJ Jr, Likert K, Higuchi D. Inhibition of factor VIIa/ tissue factor by antithrombin III and tissue factor pathway inhibitor. Blood 1993;82:1679-81.

19. Pettersen EF, Goddard TD, Huang CC, Couch GS, Greenblatt DM, Meng EC, et al. UCSF Chimera - a visualization system for exploratory research and analysis. J Comput Chem 2004; 25:1605-12.

20. Berman HM, Westbrook J, Feng Z, Gilliland G, Bhat TN, Weissig H, et al. The Protein Data Bank (www.rcsb.org). Nucleic Acids Research 2000;28:235-42.

21. Dementiev A, Dobo J, Gettins PG. Active site distortion is sufficient for proteinase inhibition by serpins: structure of the covalent complex of alpha1-proteinase inhibitor with porcine pancreatic elastase. J Biol Chem 2006;281:3452-7.

22. Dementiev A, Petitou M, Herbert JM, Gettins PG. The ternary complex of antithrombin-anhydrothrombin-heparin reveals the basis of inhibitor specificity. Nat Struct Mol Biol 2004;11: 863-7.
23. Li W, Johnson DJ, Esmon CT, Huntington JA. Structure of the antithrombin-thrombin-heparin ternary complex reveals the antithrombotic mechanism of heparin. Nat Struct Mol Biol 2004;11:857-62.

24. Bjork I, Jackson CM, Jornvall H, Lavine KK, Nordling K, Salsgiver WJ. The active site of antithrombin. Release of the same proteolytically cleaved form of the inhibitor from complexes with factor IXa, factor Xa, and thrombin. J Biol Chem 1982;257:2406-11.

25. Kojima T, Leone CW, Marchildon GA, Marcum JA, Rosenberg RD. Isolation and characterization of heparan sulfate proteoglycans produced by cloned rat microvascular endothelial cells. J Biol Chem 1992;267:4859-69.

26. de Agostini AI, Watkins SC, Slayter HS, Youssoufian H, Rosenberg RD. Localization of anticoagulantly active heparan sulfate proteoglycans in vascular endothelium: antithrombin binding on cultured endothelial cells and perfused rat aorta. J Cell Biol 1990;111:1293-304.

27. Faulk WP, Labarrere CA. Modulation of vascular antithrombin III in human cardiac allografts. Haemostasis 1993;23 Suppl 1:194-201.

28. Marciniak E. Factor-Xa inactivation by antithrombin. 3. Evidence for biological stabilization of factor Xa by factor V-phospholipid complex. Br J Haematol 1973;24:391-400.

29. Teitel JM, Rosenberg RD. Protection of factor Xa from neutralization by the heparin-antithrombin complex. J Clin Invest 1983;71:1383-91.

30. Lindhout T, Baruch D, Schoen P, Franssen J, Hemker HC. Thrombin generation and inactivation in the presence of antithrombin III and heparin. Biochemistry 1986;25:5962-9.

31. Becker DL, Fredenburgh JC, Stafford AR, Weitz JI. Exosites 1 and 2 are essential for protection of fibrin-bound thrombin from heparin-catalyzed inhibition by antithrombin and heparin cofactor II. J Biol Chem 1999;274:6226-33.

32. Kounnas MZ, Church FC, Argraves WS, Strickland DK. Cellular internalization and degradation of antithrombin IIIthrombin, heparin cofactor II-thrombin, and alpha 1-antitrypsin-trypsin complexes is mediated by the low density lipoprotein receptor-related protein. J Biol Chem 1996;271: 6523-9.

33. Olds RJ, Lane DA, Chowdhury V, De Stefano V, Leone G, Thein SL. Complete nucleotide sequence of the antithrombin gene: evidence for homologous recombination causing thrombophilia. Biochemistry 1993;32:4216-24.

34. Bock SC, Harris JF, Balazs I, Trent JM. Assignment of the human antithrombin III structural gene to chromosome 1q2325. Cytogenet Cell Genet 1985;39:67-9.

35. Olds RJ, Lane DA, Chowdhury V, Sas G, Pabinger I, Auberger $\mathrm{K}$, et al. (ATT) Trinucleotide repeats in the antithrombin gene and their use in determining the origin of repeated mutations. Hum Mutat 1994;4:31-41.

36. Prochownik EV, Orkin SH. In vivo transcription of a human antithrombin III “minigene". J Biol Chem 1984;259:1538692.

37. Aiach M, Gandrille S, Emmerich J. A review of mutations causing deficiencies of antithrombin, protein $\mathrm{C}$ and protein $\mathrm{S}$. Thromb Haemost 1995;74:81-9.

38. Tait RC, Walker ID, Perry DJ, Islam SI, Daly ME, McCall F, et al. Prevalence of antithrombin deficiency in the healthy population. Br J Haematol 1994;87:106-12.

39. McColl M, Tait RC, Walker ID, Perry DJ, McCall F, Conkie JA. Low thrombosis rate seen in blood donors and their relatives with inherited deficiencies of antithrombin and protein C: correlation with type of defect, family history, and absence of 
the factor V Leiden mutation. Blood Coagul Fibrinolysis 1996;7:689-94.

40. Mateo J, Oliver A, Borrell M, Sala N, Fontcuberta J. Laboratory evaluation and clinical characteristics of 2,132 consecutive unselected patients with venous thromboembolism - results of the Spanish Multicentric Study on Thrombophilia (EMETStudy). Thromb Haemost 1997;77:444-51.

41. Martinelli I, Mannucci PM, De Stefano V, Taioli E, Rossi V, Crosti F, et al. Different risks of thrombosis in four coagulation defects associated with inherited thrombophilia: a study of 150 families. Blood 1998;92:2353-8.

42. Heijboer H, Brandjes DP, Buller HR, Sturk A, ten Cate JW. Deficiencies of coagulation-inhibiting and fibrinolytic proteins in outpatients with deep-vein thrombosis. N Engl J Med 1990;323:1512-6.

43. De Stefano V, Finazzi G, Mannucci PM. Inherited thrombophilia: pathogenesis, clinical syndromes, and management. Blood 1996;87:3531-44.

44. Finazzi G, Barbui T. Different incidence of venous thrombosis in patients with inherited deficiencies of antithrombin III, protein C and protein S. Thromb Haemost 1994;71:15-8.

45. Vossen CY, Conard J, Fontcuberta J, Makris M, van der Meer FJ, Pabinger I, et al. Risk of a first venous thrombotic event in carriers of a familial thrombophilic defect. The European Prospective Cohort on Thrombophilia (EPCOT). J Thromb Haemost 2005;3:459-64.

46. Rosendaal FR. Risk factors for venous thrombotic disease. Thromb Haemost 1999;82:610-9.

47. Sakata T, Okamoto A, Mannami T, Matsuo H, Miyata T. Protein $\mathrm{C}$ and antithrombin deficiency are important risk factors for deep vein thrombosis in Japanese. J Thromb Haemost 2004;2:528-30.

48. Lijfering WM, Brouwer JL, Veeger NJ, Bank I, Coppens M, Middeldorp S, et al. Selective testing for thrombophilia in patients with first venous thrombosis: results from a retrospective family cohort study on absolute thrombotic risk for currently known thrombophilic defects in 2479 relatives. Blood 2009; 113:5314-22.

49. Brouwer JL, Veeger NJ, Kluin-Nelemans HC, van der Meer J. The pathogenesis of venous thromboembolism: evidence for multiple interrelated causes. Ann Intern Med 2006;145:807-15.

50. Rossi E, Za T, Ciminello A, Leone G, De Stefano V. The risk of symptomatic pulmonary embolism due to proximal deep venous thrombosis differs in patients with different types of inherited thrombophilia. Thromb Haemost 2008;99:1030-4.

51. Brouwer JL, Lijfering WM, Ten Kate MK, Kluin-Nelemans HC, Veeger NJ, van der Meer J. High long-term absolute risk of recurrent venous thromboembolism in patients with hereditary deficiencies of protein $\mathrm{S}$, protein $\mathrm{C}$ or antithrombin. Thromb Haemost 2009;101:93-9.

52. De Stefano V, Simioni P, Rossi E, Tormene D, Za T, Pagnan A, et al. The risk of recurrent venous thromboembolism in patients with inherited deficiency of natural anticoagulants antithrombin, protein C and protein S. Haematologica 2006;91: 695-8.

53. Egeberg O. Inherited antithrombin deficiency causing thrombophilia. Thromb Diath Haemorrh 1965;13:516-30.

54. Sas G, Blasko G, Banhegyi D, Jako J, Palos LA. Abnormal antithrombin III (antithrombin III "Budapest") as a cause of a familial thrombophilia. Thromb Diath Haemorrh 1974;32: $105-15$.

55. Lane DA, Bayston T, Olds RJ, Fitches AC, Cooper DN, Millar DS, et al. Antithrombin mutation database: 2nd (1997) update. For the Plasma Coagulation Inhibitors Subcommittee of the
Scientific and Standardization Committee of the International Society on Thrombosis and Haemostasis. Thromb Haemost 1997;77:197-211.

56. Lane DA, Olds RJ, Conard J, Boisclair M, Bock SC, Hultin M, et al. Pleiotropic effects of antithrombin strand $1 \mathrm{C}$ substitution mutations. J Clin Invest 1992;90:2422-33.

57. Picard V, Chen JM, Tardy B, Aillaud MF, Boiteux-Vergnes C, Dreyfus M, et al. Detection and characterisation of large SERPINC1 deletions in type I inherited antithrombin deficiency. Hum Genet 2010;127:45-53.

58. Patnaik MM, Moll S. Inherited antithrombin deficiency: a review. Haemophilia 2008;14:1229-39.

59. Kuhle S, Lane DA, Jochmanns K, Male C, Quehenberger P, Lechner K, et al. Homozygous antithrombin deficiency type II (99 Leu to Phe mutation) and childhood thromboembolism. Thromb Haemost 2001;86:1007-11.

60. Olds RJ, Lane DA, Boisclair M, Sas G, Bock SC, Thein SL. Antithrombin Budapest 3. An antithrombin variant with reduced heparin affinity resulting from the substitution L99F. FEBS Lett 1992;300:241-6.

61. Sas G, Peto I, Banhegyi D, Blasko G, Domjan G. Heterogeneity of the "classical" antithrombin III deficiency. Thromb Haemost 1980;43:133-6.

62. Martinez-Martinez I, Ordonez A, Navarro-Fernandez J, PerezLara A, Gutierrez-Gallego R, Giraldo R, et al. Antithrombin Murcia (K241E) causing antithrombin deficiency: a role for altered glycosylation. Haematologica 2010;95:1358-65.

63. Perry DJ, Daly ME, Tait RC, Walker ID, Brown K, Beauchamp NJ, et al. Antithrombin cambridge II (Ala384Ser): clinical, functional and haplotype analysis of 18 families. Thromb Haemost 1998;79:249-53.

64. Corral J, Hernandez-Espinosa D, Soria JM, Gonzalez-Conejero R, Ordonez A, Gonzalez-Porras JR, et al. Antithrombin Cambridge II (A384S): an underestimated genetic risk factor for venous thrombosis. Blood 2007;109:4258-63.

65. Finazzi G, Caccia R, Barbui T. Different prevalence of thromboembolism in the subtypes of congenital antithrombin III deficiency: review of 404 cases. Thromb Haemost 1987;58:1094.

66. Girolami A, Lazzaro AR, Simioni P. The relationship between defective heparin cofactor activities and thrombotic phenomena in AT III abnormalities. Thromb Haemost 1988;59:121.

67. Harmanci O, Ersoy O, Gurgey A, Buyukasik Y, Gedikoglu G, Balkanci F, et al. The etiologic distribution of thrombophilic factors in chronic portal vein thrombosis. J Clin Gastroenterol 2007;41:521-7.

68. Ates $\mathrm{O}$. The deficiencies of protein $\mathrm{C}$, protein $\mathrm{S}$ and antithrombin III in patients with retinal vein occlusion: a Turkish sample. Clin Lab Haematol 2006;28:391-2.

69. Calcaterra D, Martin JT, Ferneini AM, De Natale RW. Acute mesenteric and aortic thrombosis associated with antithrombin deficiency: a rare occurrence. Ann Vasc Surg 2010;24:415 e5-7.

70. Shibahara K, Tatsuta K, Orita H, Yonemura T, Kohno H. Superior mesenteric and portal vein thrombosis caused by congenital antithrombin III deficiency: report of a case. Surg Today 2007; 37:308-10.

71. Santangeli P, Sestito A. Acute left atrial thrombosis during anticoagulant therapy in a patient with antithrombin deficiency. Acta Cardiol 2008;63:635-7.

72. McColl MD, Ramsay JE, Tait RC, Walker ID, McCall F, Conkie JA, et al. Risk factors for pregnancy associated venous thromboembolism. Thromb Haemost 1997;78:1183-8.

73. Sabadell J, Casellas M, Alijotas-Reig J, Arellano-Rodrigo E, Cabero L. Inherited antithrombin deficiency and pregnancy: 
maternal and fetal outcomes. Eur J Obstet Gynecol Reprod Biol 2010;149:47-51.

74. Tu CM, Hsueg CH, Chu KM, Cheng SM, Tsao TP. Simultaneous thromboses of double coronary arteries in a young male with antithrombin III deficiency. Am J Emerg Med 2009; 27:1169 e3-6.

75. Peovska I, Maksimovic J, Kalpak O, Pejkov H, Bosevski M. Recurrent myocardial infarction in a young football player with antithrombin III deficiency. Cardiol J 2008;15:463-6.

76. Roldan V, Ordonez A, Marin F, Zorio E, Soria JM, Minano A, et al. Antithrombin Cambridge II (A384S) supports a role for antithrombin deficiency in arterial thrombosis. Thromb Haemost 2009;101:483-6.

77. Mahmoodi BK, Brouwer JL, Veeger NJ, van der Meer J. Hereditary deficiency of protein $\mathrm{C}$ or protein $\mathrm{S}$ confers increased risk of arterial thromboembolic events at a young age: results from a large family cohort study. Circulation 2008;118:1659-67.

78. Trenor CC 3rd, Michelson AD. Thrombophilia and pediatric stroke. Circulation 2010;121:1795-7.

79. Andrew M, Paes B, Milner R, Johnston M, Mitchell L, Tollefsen DM, et al. Development of the human coagulation system in the full-term infant. Blood 1987;70:165-72.

80. Andrew M, Paes B, Milner R, Johnston M, Mitchell L, Tollefsen DM, et al. Development of the human coagulation system in the healthy premature infant. Blood 1988;72:1651-7.

81. Andrew M, Vegh P, Johnston M, Bowker J, Ofosu F, Mitchell L. Maturation of the hemostatic system during childhood. Blood 1992;80:1998-2005.

82. Maclean PS, Tait RC. Hereditary and acquired antithrombin deficiency: epidemiology, pathogenesis and treatment options. Drugs 2007;67:1429-40.

83. Bushman JE, Palmieri D, Whinna HC, Church FC. Insight into the mechanism of asparaginase-induced depletion of antithrombin III in treatment of childhood acute lymphoblastic leukemia. Leuk Res 2000;24:559-65.

84. Hernandez-Espinosa D, Minano A, Martinez C, Perez-Ceballos E, Heras I, Fuster JL, et al. L-asparaginase-induced antithrombin type I deficiency: implications for conformational diseases. Am J Pathol 2006;169:142-53.

85. Hernandez-Espinosa D, Minano A, Ordonez A, Mota R, Martinez-Martinez I, Vicente V, Corral J. Dexamethasone induces a heat-stress response that ameliorates the conformational consequences on antithrombin of $\mathrm{L}$-asparaginase treatment. J Thromb Haemost 2009;7:1128-33.

86. Abildgaard U, Lie M, Odegard OR. Antithrombin (heparin cofactor) assay with "new" chromogenic substrates (S-2238 and Chromozym TH). Thromb Res 1977;11:549-53.
87. Odegard OR, Lie M, Abildgaard U. Antifactor Xa activity measured with amidolytic methods. Haemostasis 1976;5: 265-75.

88. Meijer P, Kluft C, Haverkate F, De Maat MP. The long-term within- and between-laboratory variability for assay of antithrombin, and proteins $\mathrm{C}$ and $\mathrm{S}$ : results derived from the external quality assessment program for thrombophilia screening of the ECAT Foundation. J Thromb Haemost 2003;1:748-53.

89. Demers C, Henderson P, Blajchman MA, Wells MJ, Mitchell L, Johnston M, et al. An antithrombin III assay based on factor Xa inhibition provides a more reliable test to identify congenital antithrombin III deficiency than an assay based on thrombin inhibition. Thromb Haemost 1993;69:231-5.

90. Conard J, Bara L, Horellou MH, Samama MM. Bovine or human thrombin in amidolytic at III assays. Influence of heparin cofactor II. Thromb Res 1986;41:873-8.

91. Rossi E, Chiusolo P, Za T, Marietti S, Ciminello A, Leone G, et al. Report of a novel kindred with antithrombin heparinbinding site variant (47 Arg to His): demand for an automated progressive antithrombin assay to detect molecular variants with low thrombotic risk. Thromb Haemost 2007;98:695-7.

92. Parvez Z, Fareed J, Messmore HL, Moncada R. Laser nephelometric quantitation of antithrombin-III (AT-III) development of a new assay. Thromb Res 1981;24:367-77.

93. Von Kaulla E, Von Kaulla KN. Antithrombin 3 and diseases. Am J Clin Pathol 1967;48:69-80.

94. Marciniak E, Gockerman JP. Heparin-induced decrease in circulating antithrombin-III. Lancet 1977;ii:581-4.

95. Conard J. Inhibitors of blood coagulation. In: Thompson JM, editor. Blood Coagulation and Haemostasis, Vol. 2nd ed. Edinburgh: Churchill Livingstone, 1980:200-21.

96. Laffan MaM R. Investigation of a thrombotic tendency. In: Lewis SM, Bain BJ, Bates I, editors. Practical Haematology, Vol. 10th ed. Philadelphia: Churchill Livingstone, 2006:44163.

97. Andersson G, Fagrell B, Holmgren K, Johnsson H, Ljungberg B, Wilhelmsson S. Antithrombin III in patients with acute deep vein thrombosis during heparin treatment (subcutaneous and intravenous) and during and after treatment with oral coumarins. Thromb Res 1984;34:333-40.

98. O'Brien JR, Etherington MD. Effect of heparin and warfarin on antithrombin III. Lancet 1977;2:1231.

99. Kottke-Marchant K, Duncan A. Antithrombin deficiency: issues in laboratory diagnosis. Arch Pathol Lab Med 2002; 126:1326-36.

100. Conard J. Antithrombin activity and antigen. In: Jespersen J, Bertina RM, Haverkate F, editors. Laboratory Techniques in Thrombosis A Manual 2nd Revised Edition of the ECAT Assay Procedures. Dordrecht: Kluwer, 1999:121-8. 